Este obra forma parte del acervo de la Biblioteca Jurídica Virtual del Instituto de Investigaciones Jurídicas de la UNAM www.juridicas.unam.mx

\title{
LA eVALUACIÓN DE LAS NUEVAS REgLAS DE FINANCIAMIENTO ELECTORAL A LA LUZ DE LA EXPERIENCIA DEL PROCESO ELECTORAL 2015
}

\author{
Oswaldo Chacón Rojas ${ }^{1}$
}

\section{Sumario}

I. Las reglas del financiamiento político-electoral en México.

II. La evaluación del funcionamiento de las reglas en las elecciones federales 2015.

III. Propuestas de fortalecimiento.

\section{LAS REGLAS DEL FINANCIAMIENTO POLÍTICO-ELECTORAL EN MÉXICO}

La influencia del exceso de dinero o de origen oscuro en la renovación periódica de los cargos públicos continúa siendo una de las preocupaciones centrales de las democracias contemporáneas. No es casualidad que en los últimos años diversas instituciones y agencias internacionales hayan destinado importantes esfuerzos a la realización de estudios comparativos en el mundo con el fin de diagnosticar debidamente el problema, como los desarrollados por la Organización de Estados Americanos (OEA), ${ }^{2}$ el Instituto Internacional para la Democracia y Asistencia Electoral (IDEA), ${ }^{3} 0$ la Fundación

\footnotetext{
$1 \mathrm{UNACH}$.

2 Manual para las Misiones de Observación del financiamiento político de la OEA, disponible en:

http://www.oas.org/es/sap/deco/publicaciones.asp.

3 Political Finance Regulations around the World. An Overview of the International IDEA Database, disponible en: http://www.idea.int/publications/political-finance-

regulations/loader.cfm?csModule=security/getfile\&pageid $=52121$.
} 
Este obra forma parte del acervo de la Biblioteca Jurídica Virtual del Instituto de Investigaciones Jurídicas de la UNAM www.juridicas.unam.mx

Internacional para Sistemas Electorales (IFES). ${ }^{4}$ Lo cierto es que dichos estudios son básicamente descriptivos de los modelos existentes en cada país, pero no hay un consenso teórico ni político respecto de un modelo a seguir. La adopción de los modelos de financiación político-electoral depende de las tradiciones constitucionales y contexto de cada país, aunque sí hay una tendencia hacia la promoción de aquellos que estimulen los recursos que favorecen la igualdad de condiciones, como son los fondos públicos, la restricción de aquellos que la perjudican, como son los fondos privados, la limitación de los gastos de campaña, y el derecho a la información y la transparencia.

El modelo de financiamiento político-electoral mexicano está acorde con estas recomendaciones. El financiamiento de los partidos es predominantemente público, para actividades ordinarias, específicas y de campaña, y es distribuido por la autoridad electoral administrativa - Instituto Nacional Electoral (INE) - entre los partidos, de acuerdo a la regla 30-70. Es decir, el 30\% de los mismos se distribuye en partes iguales, y el $70 \%$ de acuerdo a los resultados electorales obtenidos en la elección de diputados inmediata anterior -artículo 41, base II de la Constitución Política (CPEUM) y artículo 50.1 de la Ley General de Partidos Políticos (LGPP)—, mientras que esta última modalidad de financiación está sujeta a topes restrictivos, y todo el sistema está sujeto a controles exhaustivos realizados por un organismo autónomo, técnico y especializado como es el INE. Este modelo ha cumplido con sus objetivos en la medida en que ha facilitado el fortalecimiento de un sistema plural de partidos, donde diversas fuerzas políticas acceden a importantes recursos económicos, y bajo marcadas restricciones de gasto que les ha permitido competir en situaciones de igualdad.

No obstante, la reforma constitucional de 2014 contempló modificaciones a las reglas en materia de financiamiento, partiendo del diagnóstico de que el modelo adolece de ciertas deficiencias y limitaciones que deben corregirse. Con la reforma se buscó fortalecer el modelo frente a la presumible existencia de un mercado de financiación informal e ilegal en las campañas, derivado de la sensación de que las acciones u omisiones de la autoridad electoral dejaron en los partidos opositores, sobre todo en las investigaciones de los casos de las tarjetas Monex o Soriana, o la presumible financiación

\footnotetext{
4 Political Finance Regulation: The Global Experiencie, disponible en:

http://Www.ifes.org/ /media/Files/Publications/Books/2009/Political\%20Finance\%20Regulation\%20The\%20G Iobal\%20Experience/Political_Finance_Regulation_The_Global_Experience.pdf.
} 
Este obra forma parte del acervo de la Biblioteca Jurídica Virtual del Instituto de Investigaciones Jurídicas de la UNAM

ilegal de autoridades gubernamentales locales, o frente a la financiación de actividades clientelares. Los puntos más importantes de la reforma fueron los siguientes:

a) A efecto de prevenir la demanda fondos ilegales, las nuevas reglas mantuvieron la fórmula de financiamiento para partidos nacionales, pero aumentaron el dinero de los partidos en el ámbito estatal, homologando la fórmula de integración en los estados a la nacional, $65 \%$ del salario mínimo multiplicado por el número de ciudadanos inscritos en el padrón electoral del estado de que se trate (artículo 116, fracción IV, inciso g de la CPEUM). Lo anterior, toda vez que en México los partidos políticos también tienen derecho a recibir recursos de los estados. Como en la mayoría de las entidades, el factor que se usaba para determinar la bolsa de partidos era menor a $65 \%$ del salario mínimo —en muchas era de $20 \%$-, el resultado fue un incremento de $52 \%$ en el financiamiento a los partidos en el ámbito estatal.

b) Con el propósito de fomentar la transparencia, se quitó la barrera a los topes generales de aportaciones privadas, las cuales casi nunca son reportados por los partidos. La reforma elimina el tope máximo anual de $10 \%$ de financiamiento privado para permitirles mayores ingresos. De esta manera, las aportaciones de candidatos, así como de simpatizantes durante los procesos electorales, destinadas a ser utilizadas en las campañas, no pueden superar el $10 \%$ del tope de gasto para la elección presidencial inmediata anterior (artículo 56.2, inciso b) de la LGPP). Se introdujo una fórmula de topes máximos anuales por cada tipo de aportación que recibirán los partidos políticos, a diferencia de la legislación electoral anterior que señalaba claramente un tope al dinero privado que podría ingresar a los partidos políticos, equivalente a $10 \%$ del financiamiento público total. La ley vigente solamente pone un límite a las aportaciones que en lo individual pueden hacer los simpatizantes a su partido, pero no señala un límite para la totalidad de los simpatizantes. El único acotamiento que sería aplicable, en este caso, es el principio general que rige el financiamiento de los partidos políticos, que es la preponderancia del dinero público sobre el privado. El financiamiento individual de simpatizantes ahora tiene un tope individual. El tope de financiamiento de militantes es el $2 \%$ del financiamiento público total otorgado a la totalidad de los partidos políticos (el numeral dos del artículo 56 de la LGPP).

c) Para distribuir la bolsa solamente entre partidos que gocen de una importante representación, se incrementa el umbral para acceder al financiamiento público. 
Este obra forma parte del acervo de la Biblioteca Jurídica Virtual del Instituto de Investigaciones Jurídicas de la UNAM

Ahora los partidos tendrán que haber obtenido por lo menos el 3\% de la votación válida emitida, antes era suficiente el $2 \%$ (artículo 41, base I, de la CPEUM). Además, la cantidad de votos necesaria para acceder a las prerrogativas se eleva aún más con el cambio en la base sobre la cual se calcula: anteriormente era el $2 \%$ de la votación total emitida, mientras que la reforma lo establece en el 3\% de la votación válida emitida (artículo 41, base I, de la CPEUM).

d) Se homogenizan los sistemas de contabilidad partidista. El decreto de la reforma constitucional obligó al legislativo a incluir en la ley de partidos los lineamientos homogéneos de contabilidad, la cual debe ser pública y de acceso por medios electrónicos (segundo transitorio, fracción I, inciso g), punto 2 de la CPEUM). Por ello, la LGPP establece un nuevo régimen de contabilidad aplicable a todos los partidos políticos (artículo 59).

e) Se introduce un sistema de fiscalización en línea, concurrente al desarrollo de las precampañas y campañas. A fin de evitar los rebases en los topes de gastos de campaña, o el "maquillaje" de cifras, el INE se encuentra obligado a desarrollar un Sistema de Contabilidad en Línea, que obliga a partidos, coaliciones, aspirantes, precandidatos, candidatos y candidatos independientes, a entregar su contabilidad diaria de los gastos de precampaña y campaña. El sistema de contabilidad se lleva a cabo a través de un sistema informático, donde los partidos realizan sus registros en línea. El INE tiene el acceso irrestricto a toda la información contenida en los registros contables de los partidos (artículo 60.2 de la LGPP). Con ello, se impone a los partidos la obligación de entregar al Instituto sus estados financieros en un plazo de 72 horas, con un corte al momento de la solicitud (artículo 61.1, inciso f) de la LGPP). Los partidos deberán registrar a diario: registros contables donde identifiquen cada operación, relacionándola con la documentación comprobatoria, adquisiciones de activo fijo realizadas, distinguiendo entre los adquiridos y los recibidos en aportación o donación de un tercero, con documentación que permita identificar la fecha de adquisición o alta del bien, sus características físicas.

f) Los candidatos y precandidatos se vuelven responsables solidarios del cumplimiento de los informes de campaña y precampaña; en caso de sanciones, se analizará de manera separada las infracciones en que incurran (artículo 79.1, inciso a), fracciones I al IV de la LGPP). 
Este obra forma parte del acervo de la Biblioteca Jurídica Virtual del Instituto de Investigaciones Jurídicas de la UNAM www.juridicas.unam.mx

g) Disminuyen los plazos de presentación de informes financieros. Los informes de precampaña deberán ser presentados por los partidos políticos para cada uno de los precandidatos a candidatos a cargo de elección popular, registrados para cada tipo de precampaña, especificando el origen y monto de los ingresos, así como los gastos realizados, a más tardar dentro de los diez días siguientes al de la conclusión de las precampañas. La Unidad Técnica de Fiscalización tendrá 15 días para la revisión de dichos informes. En caso de encontrar errores u omisiones, la Unidad avisará al partido político para que éste, en el término de siete días contados a partir de dicha notificación, presente las aclaraciones o rectificaciones que considere pertinentes. Una vez concluidos los plazos, la Unidad contará con un término de diez días para emitir el dictamen consolidado, así como el proyecto de resolución respectivo y para someterlo a consideración de la Comisión de Fiscalización. La Comisión contará con seis días para aprobar los proyectos y, una vez aprobados, deberá presentarlos ante el Consejo General en un plazo de 72 horas. El Consejo General contará con un plazo de seis días para su discusión y aprobación (artículo 79.1, inciso c) de la LGPP). Por su parte, los informes de campaña deberán ser presentados por los partidos políticos, para cada una de las campañas en las elecciones respectivas, especificando los gastos que el partido político y el candidato hayan realizado en el ámbito territorial correspondiente. Los partidos políticos presentarán informes de ingresos y gastos por periodos de treinta días, contados a partir de que dé inicio la etapa de campaña, los cuales deberán entregar a la Unidad Técnica dentro de los siguientes tres días de concluido cada periodo. Para los informes de campaña, la Unidad Técnica deberá revisar y auditar, simultáneamente al desarrollo de la campaña, el destino que le den los partidos políticos a los recursos de campaña. Para ello, contará con diez días para revisar la documentación soporte y la contabilidad presentada con cada informe parcial. En el caso de la existencia de errores u omisiones técnicas en la documentación soporte y contabilidad presentada, se otorgará un plazo de cinco días (contados a partir de la notificación), para que el partido presente las aclaraciones o rectificaciones que considere pertinentes. Una vez concluida la revisión del último informe, la Unidad Técnica contará con un término de diez días para realizar el dictamen consolidado y la propuesta de resolución, así como para someterlos a consideración de la Comisión de Fiscalización. La Comisión tendrá un término de seis días para votar dichos proyectos y presentarlos al Consejo General, y éste debe votarlos en un término improrrogable de seis días (artículo 79.1, inciso d) de la LGPP). 
Este obra forma parte del acervo de la Biblioteca Jurídica Virtual del Instituto de Investigaciones Jurídicas de la UNAM www.juridicas.unam.mx

Los partidos políticos podrán impugnar ante el Tribunal el dictamen consolidado. En ese caso, el Consejo General del Instituto deberá remitir al Tribunal el dictamen consolidado de la Unidad Técnica y el informe respectivo (artículo 82 de la LGPP).

h) La centralización de la fiscalización. El artículo 41, base V, apartado B, penúltimo párrafo, establece que el INE será encargado de realizar la fiscalización y vigilancia durante la campaña, del origen y destino de todos los recursos de los partidos y candidatos.

i) Se establece, vía determinación de la Suprema Corte de Justicia de la Nación (SCJN), que los gastos de operatividad electoral deben ser considerados de campaña. La ley señalaba que los gastos para su operación ordinaria, para el cumplimiento de sus obligaciones estatutarias y para el sostenimiento de sus órganos directivos y de sus organizaciones durante los procesos electorales, no se considerarán dentro de los gastos de campaña (artículo 76.2 de la LGPP). Asimismo, que todos los bienes o servicios que se destinen a la campaña deberán tener como propósito directo la obtención del voto en las elecciones federales o locales, con la única excepción del gasto relativo a estructuras electorales que, como se vio en el apartado anterior, se considera parte del gasto ordinario (artículo 76.3 de la LGPP). Estas disposiciones fueron invalidadas por la SCJN el 4 de septiembre de 2014. La Corte invalidó a los partidos políticos disfrazar los recursos públicos que financian campañas electorales como gasto ordinario. Los ministros anularon diversas fracciones del artículo 72 de la LGPP, que clasificaba como gasto ordinario el dinero destinado a estructura partidista de campaña y a los relativos a estructuras electorales que participan en dicho proceso. Los ministros resolvieron que este tipo de gastos no están previstos en la Constitución, pues además buscan evitar la fiscalización de gastos de campaña, con lo que rompen el principio de separarlos de los gastos de sostenimiento cotidiano y permanente de los partidos.

j) Se establecen reglas para dar certidumbre al prorrateo de gastos. Los gastos genéricos de campaña son los realizados en actos de campaña y de propaganda en la que el partido o la coalición promueva o invite a votar por un conjunto de candidatos a cargos de elección popular que postulen, siempre y cuando no se especifique el candidato o el tipo de campaña; cuando no se identifique algún candidato o tipo de campaña, pero se difunda alguna política pública o propuesta del partido o coalición, y cuando se publique o difunda el emblema o la mención de lemas con los que se 
Este obra forma parte del acervo de la Biblioteca Jurídica Virtual del Instituto de Investigaciones Jurídicas de la UNAM www.juridicas.unam.mx

identifique al partido, coalición o sus candidatos o los contenidos de sus plataformas electorales (artículo 83.1 de la LGPP). La nueva legislación termina con la flexibilidad en distribuir los gastos entre varios candidatos promocionados a través del mismo elemento de propaganda, y establece reglas estrictas de prorrateo (artículo 83.2, incisos a) al k) de la LGPP).

k) Se establece el reconocimiento constitucional de la causal de nulidad de elección por rebase de tope de gastos de campaña o por haber recibido recursos de procedencia ilícita. El artículo 41, base VI, de la Constitución establece que será causal de nulidad de la elección el rebase del tope de gasto de campaña en un $5 \%$ del monto total autorizado, o el haber recibido o utilizado recursos de procedencia ilícita o recursos públicos en las campañas. Se determina que dichas violaciones tendrán que acreditarse de manera objetiva y ser determinantes para el resultado electoral. La misma Constitución señala que se considerarán determinantes cuando la diferencia entre los candidatos en el primer y segundo lugar sea menor al 5\% (artículo 41, base VI de la CPEUM y artículo 78 bis, 2 de la Ley General del Sistema de Medios de Impugnación en Materia Electoral - LGSMIME-). También se establece que las elecciones federales o locales serán nulas por violaciones graves, dolosas y determinantes (artículo 78 bis, 1 de la LGSMIME). Se considerarán como violaciones graves aquellas conductas irregulares que produzcan una afectación sustancial a los principios constitucionales en la materia y pongan en peligro el proceso electoral y sus resultados (artículo 78 bis, 4 de la LGSMIME). Asimismo, se calificarán como dolosas aquellas conductas realizadas con pleno conocimiento de su carácter ilícito, llevadas a cabo con la intención de obtener un efecto indebido en los resultados del proceso electoral (artículo 78 bis, 5 de la LGSMIME).

I) Se establecieron reglas de financiación a candidaturas independientes.

\section{LA EVALUACIÓN DEL FUNCIONAMIENTO DE LAS REGLAS EN LAS ELECCIONES FEDERALES 2015}

En teoría, la reforma viene a robustecer el modelo de financiación electoral de amplio predominio de fondos públicos, restricciones a las aportaciones privadas y exhaustivos mecanismos de control de esta financiación formal político-electoral, a fin de contar con contiendas más transparentes, equitativas y desvinculadas del factor distorsionador del dinero ilícito. Una reforma adicional al robusto andamiaje normativo tendría hacia la 
Este obra forma parte del acervo de la Biblioteca Jurídica Virtual del Instituto de Investigaciones Jurídicas de la UNAM

perfección del modelo, lo cual muy rápidamente enfrentaría su prueba de fuego con las elecciones legislativas de 2015 y elecciones locales concurrentes en 16 estados. La evaluación de la reforma pasa por verificar si el modelo cumplió o no su objetivo de satisfacer las demandas de fondos de los partidos y candidatos, si favoreció la transparencia y rendición de cuentas del mercado de financiación, y si mejoró los mecanismos de prevención, control y sanción de conductas ilícitas.

Este ejercicio de evaluación precisa modificar ciertos paradigmas que han dominado los estudios en torno al modelo de financiación político-electoral en nuestro país. En efecto, en México el análisis se ha reducido básicamente a la verificación de los resultados electorales, puesto que fue diseñado para permitir el tránsito de un régimen de partido hegemónico hacia uno que permitiera la competencia equitativa de diversas fuerzas partidistas. En consecuencia, si los resultados favorecían el pluripartidismo, luego entonces teníamos elementos para suponer que el modelo estaba funcionando. Bajo este supuesto de que las reglas funcionan en la medida que tiendan al fortalecimiento del sistema de partidos, se trataba que éstos contaran con el mayor presupuesto posible, y que fuesen incomodados lo menos posible en su ejercicio.

Una vez que el pluripartidismo se ha ido consolidando, la evaluación del funcionamiento de las reglas debe atender otros factores. Se requiere ir más allá de la mera verificación de resultados electorales, y transitar al análisis de cómo se generan esos resultados. La evaluación requiere la construcción de nuevos indicadores que permitan medir factores como el nivel de transparencia, o la financiación durante todo el proceso, por ejemplo. En conclusión, nuestra tesis es que la evaluación de las reglas, en función de los resultados electorales que generan, resulta insuficiente para saber que un sistema de financiación está fomentando la equidad y la transparencia.

Desde esta perspectiva, un instrumento de investigación que resulta pertinente es el análisis de la información contenida en la verificación del cumplimiento de las reglas de financiación político-electoral contenida en los dictámenes de fiscalización llevado a cabo por la propia autoridad electoral. Recurrir a los datos contenidos en estos documentos nos permite llevar a cabo estudios, tomando como base datos objetivos, sin caer en estridencias; asimismo, conocer mayores elementos detrás del uso, instrumentación y alcances de las reglas. En los dictámenes encontramos los informes financieros presentados por los partidos, los reportes del sistema en línea, los resultados de los procedimientos de fiscalización llevados a cabo por la Unidad Técnica, las observaciones 
Este obra forma parte del acervo de la Biblioteca Jurídica Virtual del Instituto de Investigaciones Jurídicas de la UNAM www.juridicas.unam.mx

realizadas por la Comisión de Fiscalización, las resoluciones aprobadas por el Consejo General del INE, entre otras cosas.

Para este estudio, analizamos los dictámenes de fiscalización que fueron aprobados por la Comisión de Fiscalización y el Consejo General del INE el 20 de julio de 2015.

De dicho estudio concluimos que aun y cuando el modelo se ha robustecido, atajando disparadores de gasto, acompañado de nuevas acciones de control, de investigación y colaboración interinstitucional, que han sido aplicados a cabalidad por la autoridad electoral, como la revisión documental de ingresos y egresos, la observación, inspección, o fiscalización en terreno, las verificaciones in locuo, el monitoreo de propaganda callejera, los cotejos, confirmaciones de operaciones con terceros, el acceso a información bancaria y fiscal, el requerimiento de información a personas físicas y morales, o el intercambio de información con otras autoridades, éste no desincentiva el mercado de la financiación informal, y sigue adoleciendo de dificultades para detectarlo y prevenirlo. El análisis arroja los siguientes hallazgos:

1. La bolsa de fondos públicos se incrementó considerablemente, producto de la obligación en los estados de homologar la fórmula para integrarla, con lo que los partidos políticos (PP) obtuvieron mayor financiamiento para sus actividades de campaña de los que necesitaban (tabla 1). La cifra total de financiamiento público del ejercicio 2015 para actividades ordinarias permanentes de todos los partidos políticos nacionales ascendió a \$3,909,545,803.15 (tres mil novecientos nueve millones quinientos cuarenta $y$ cinco mil ochocientos tres pesos 15/100 M. N.), $\$ 1,172,863,740.95$ (mil ciento setenta y dos millones ochocientos sesenta y tres mil setecientos cuarenta pesos 95/100 M. N.), por concepto de financiamiento para gastos de campañas; y la cifra del financiamiento público para actividades específicas, correspondientes a la educación, capacitación, investigación socioeconómica y política, así como a las tareas editoriales en 2015 , es de $\$ 106,730,600.43$ (ciento seis millones setecientos treinta mil seiscientos pesos $43 / 100 \mathrm{M}$. N.).

En los últimos seis años, el total de financiamiento federal que reciben los partidos se ha incrementado en $\mathbf{2 1 . 4 \%}$ en términos reales. Bajo esta fórmula, México está entre los países que más recursos destinan a financiar su sistema electoral. Según la IFES, el promedio del financiamiento público en México fue 18 veces superior al de los países de América Latina en el periodo 2001-2004. Aunque la reforma de 2014 prometió 
Este obra forma parte del acervo de la Biblioteca Jurídica Virtual del Instituto de Investigaciones Jurídicas de la UNAM www.juridicas.unam.mx

"abaratar" el costo de las elecciones, lo que se observa es un incremento constante para financiar a los partidos. Esa inversión es justificable en la medida que cumple con el principal objetivo planteado cuando se diseñó el sistema en los años noventa, la equidad en la competencia entre partidos, algo que se ha conseguido indiscutiblemente, pues la brecha entre partidos se redujo significativamente, y ello contribuyó al mayor pluralismo y la alternancia que se ha dado desde la presidencia de la República hasta gobiernos estatales y municipales. Sin embargo, la misma equidad podría lograrse con una nivelación a la baja si los recursos de todos los partidos fueran menores. Además, que todos los partidos tengan la posibilidad de ganar, no significa que el dinero ha dejado de ser un factor de desventaja o inequidad en esos triunfos. Por ello, estamos obligados a evaluar el modelo con base a otros indicadores.

\section{TABLA 1}

\begin{tabular}{|c|r|r|r|r|}
\hline PP & MINISTRACION & FONDOS PUBLICOS REPORTADOS & \multicolumn{1}{|c|}{ DIFERENCIA } \\
\hline PAN & $257,623,000$ & $222,856,791$ & $34,766,209$ \\
\hline PRI & $306,726,482$ & $44,528,100$ & $262,198,382$ \\
\hline PRI-PVEM & $327,603,726$ & $171,609,034$ & $155,994,692$ \\
\hline PVEM & $96,970,155$ & $31,564,811$ & $65,405,344$ \\
\hline PRD & $196,394,734$ & $116,390,947$ & $80,003,787$ \\
\hline PT & $84,586,629$ & $80,905,613$ & $3,681,016$ \\
\hline PRD-PT & & $61,214,901.89$ & $-12,020,694$ \\
\hline MC & $79,773,722$ & $91,794,416$ & $2,778,647$ \\
\hline NA & $80,416,725$ & $77,638,078$ & $5,954,138$ \\
\hline PH & $23,457,274$ & $17,503,136$ & $9,797,065$ \\
\hline P.M. & $23,457,274$ & $13,660,209$ & $4,395,410$ \\
\hline PES & $23,457,274$ & $19,061,864$ & \\
\hline
\end{tabular}

2. Diferencias entre lo reportado en el sistema en línea y lo presentado en los informes financieros refleja problemas en la integración de los informes y de entendimiento del sistema, pero también la baja calidad de los reportes.

TABLA 2 
Este obra forma parte del acervo de la Biblioteca Jurídica Virtual del Instituto de Investigaciones Jurídicas de la UNAM www.juridicas.unam.mx

DOI: http://dx.doi.org/10.22201/iij_.24487910e.2016.9.10102

\begin{tabular}{|c|r|r|r|}
\hline PP & $\begin{array}{c}\text { TOTAL DE INGRESOS } \\
\text { REPORTADOS POR EL PP }\end{array}$ & $\begin{array}{c}\text { TOTAL DE INGRESOS CON } \\
\text { VERIFICACIÓN INE }\end{array}$ & \multicolumn{1}{c|}{ DIFERENCIAL } \\
\hline PAN & $170,629,611$ & $231,234,306.93$ & $60,604,695$ \\
\hline PRI & $5,773,528$ & $46,338,417.95$ & $40,564,889$ \\
\hline PRI-PVEM & $130,049,290$ & $178,017,169.45$ & $47,967,879$ \\
\hline PVEM & $13,512,544$ & $31,564,811.00$ & $18,052,267$ \\
\hline PRD & & $128,722,792.70$ & \\
\hline PT & $79,069,590$ & $81,135,487.65$ & $2,065,897$ \\
\hline PRD-PT & & $69,771,626.05$ & \\
\hline MC & & $97,731,376.37$ & $35,759,003$ \\
\hline NA & $45,036,772$ & $80,795,775.86$ & $17,429,003$ \\
\hline PH & & $21,328,850.59$ & $15,714,835$ \\
\hline P.M. & $9,902,731$ & $\$ 27,331,734.11$ & \\
\hline PES & $23,599,420$ & $39,314,255.74$ & \\
\hline
\end{tabular}

3. A pesar de que se liberaron los topes a las aportaciones privadas, éstas siguen sin declararse de manera importante. Continúan predominando las subdeclaraciones y el ocultamiento de información. El ente fiscalizador presenta problemas para acreditar aportaciones privadas no reportadas.

TABLA 3

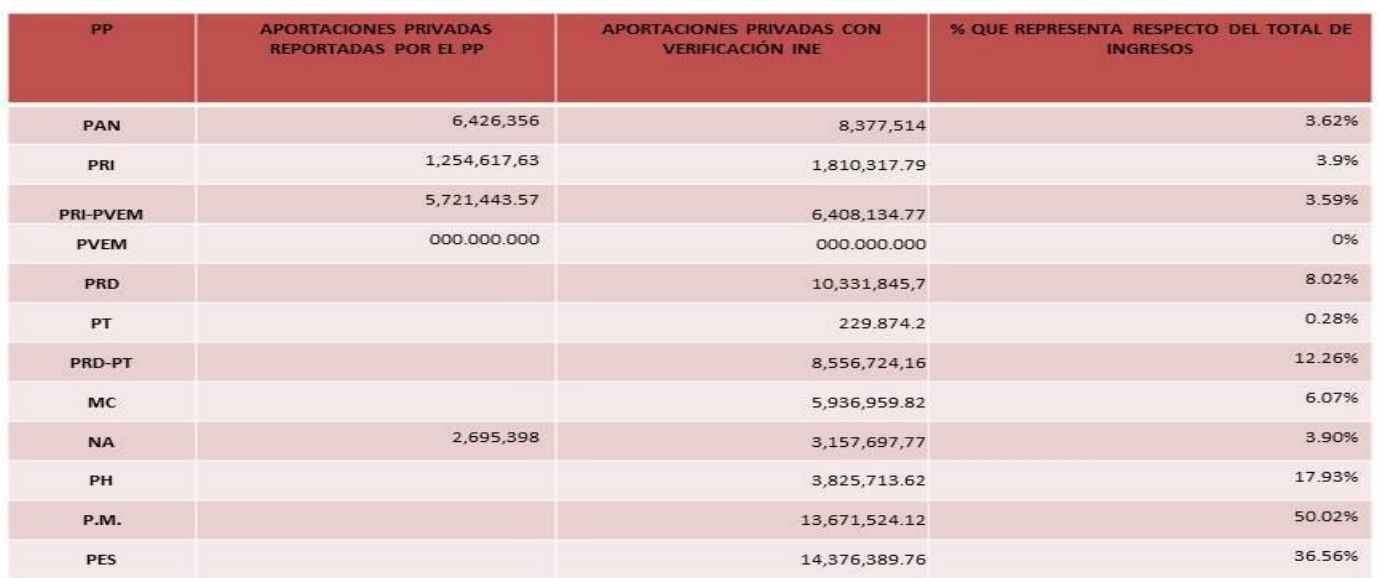

\section{1}

DR @ 2018. Instituto de Investigaciones Jurídicas, UNAM, https://revistas.juridicas.unam.mx/index.php/derecho-electoral/issue/archive 
Este obra forma parte del acervo de la Biblioteca Jurídica Virtual del Instituto de Investigaciones Jurídicas de la UNAM www.juridicas.unam. $\mathrm{mx}$

4. Los PP presentaron distintas versiones de sus informes, restando credibilidad a los mismos. Diferencias entre primer y segundo reporte; el nivel de especificidad en la información y el detalle exigido en los formatos de informes no ha logrado incidir en la calidad de la información, ya que es entregada con faltas a los lineamientos y reglas establecidas; el alto número de versiones de informes de los PP, producto de las observaciones efectuadas por la UF, con un promedio de 6.1428 versiones, determina que de inicio la información presentada no es confiable.

TABLA 4

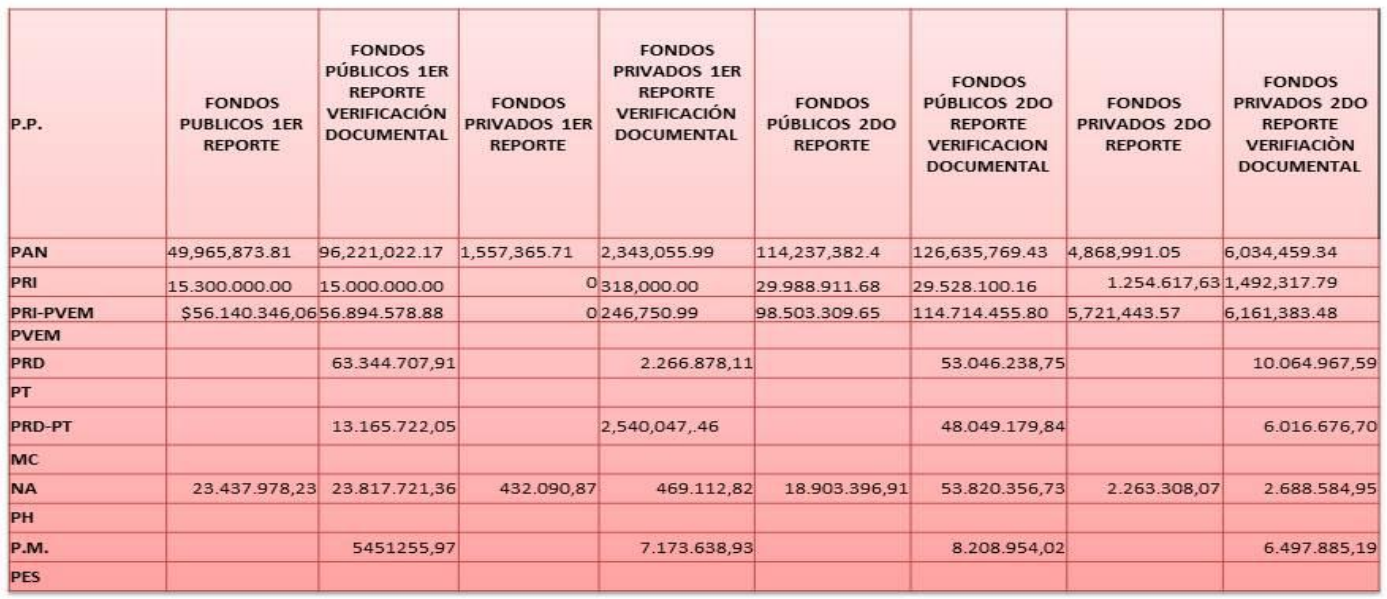

5. Los PP son poco proclives a reportar gastos. Predominan las subdeclaraciones en los gastos y el ocultamiento de información. Presentan distintas versiones restando credibilidad a su información; la variación de las cifras reportadas por los PP en sus ingresos es considerable, pero en los egresos se observa una variación aún mayor; los egresos sin reportar se van acreditando conforme aumenta el número de versiones de informes, lo que demuestra que los PP únicamente reportan gastos en función de lo que la UF les acredita, por lo que se puede presumir que la información que presentan no es suficiente ni confiable.

\section{TABLA 5}


Este obra forma parte del acervo de la Biblioteca Jurídica Virtual del Instituto de Investigaciones Jurídicas de la UNAM www.juridicas.unam.mx https://biblio.juridicas.unam.mx/bjv

https://revistas.juridicas.unam.mx/

DOI: http://dx.doi.org/10.22201/iij.24487910e_2016.9.10102

\begin{tabular}{|c|c|c|c|}
\hline P.P. & $\begin{array}{l}\text { TOTAL DE FONDOS } \\
\text { PÚBLICOS REPORTADOS }\end{array}$ & $\begin{array}{l}\text { TOTAL DE FONDOS PRIVADOS } \\
\text { REPORTADOS }\end{array}$ & INGRESOS TOTALES REPORTADOS \\
\hline PAN & $222,856,791.6$ & $8,377,515.33$ & $231,234,306.93$ \\
\hline PRI & $44,528,100.16$ & $1,810,317.79$ & $46,338,417.95$ \\
\hline PRI-PVEM & $171,609,034.68$ & $6,408,134.77$ & $178,017,169.45$ \\
\hline PVEM & $31,564,811.00$ & 0 & $31.564 .811,00$ \\
\hline PRD & 116.390 .947 & $10.331 .845,70$ & $128.722 .792,70$ \\
\hline PT & $80.905 .613,45$ & 229.874 .2 & $81.135 .487,65$ \\
\hline PRD-PT & $61.214 .901,89$ & $8.556 .724,16$ & $69.771 .626,05$ \\
\hline MC & $91.794 .416,55$ & $5.936 .959,82$ & $97,731,376.37$ \\
\hline NA & $77.638 .078,09$ & $3.157 .697,77$ & $80,795,775.86$ \\
\hline PH & $17.503 .136,97$ & $3.825 .713,62$ & $21.328 .850,59$ \\
\hline P.M. & $13.660 .209,99$ & $13.671 .524,12$ & $\$ 27,331,734.11$ \\
\hline PES & $19,061,864$ & $20,252,391$ & $39,314,255.74$ \\
\hline
\end{tabular}

6. El ente fiscalizador presenta problemas para acreditar gastos que no son reportados. Son muy pocas las observaciones de gasto no atendidas.

TABLA 6

\begin{tabular}{|c|c|c|c|c|c|c|}
\hline P.P. & GASTO 1ER INFORME & $\begin{array}{l}\text { GASTO 1ER } \\
\text { INFORME } \\
\text { VERIFICACION } \\
\text { DOCUEMENTAL }\end{array}$ & $\begin{array}{l}\text { GASTO } 2 \text { DO } \\
\text { INFORME }\end{array}$ & $\begin{array}{l}\text { GASTO } 2 \text { DO } \\
\text { INFORME } \\
\text { VERIFICACION } \\
\text { DOCUEMENTAL }\end{array}$ & $\begin{array}{l}\text { GASTO TOTAL } \\
\text { REPORTADO }\end{array}$ & $\begin{array}{c}\text { GASTO } \\
\text { REPORTADO } \\
\text { VERIFICADO POR } \\
\text { EL INE } \\
\end{array}$ \\
\hline PAN & $37,996,716.30$ & $90,808,826.21$ & 121957520.51 & 134831518.97 & $159,954,236$ & $225,640,345.18$ \\
\hline PRI & $8,869,853$ & $8.967 .853,80$ & $31,902,887$ & $36,077,362.01$ & $40,772,740$ & $\$ 45,045,215.81$ \\
\hline PRI-PVEM & 28235296.66 & $31,105,885.82$ & 107801520.06 & $141,721,649.71$ & & $172,827,535$ \\
\hline PVEM & $5,627,727.37$ & $11.956 .778,92$ & $13,758,962.38$ & $19.614 .951,45$ & $19,386,689$ & $31.571 .730,37$ \\
\hline PRD & & $53.001 .734,62$ & & $70.804 .613,74$ & & $123.806 .348,36$ \\
\hline PT & & & & & & $67.745 .791,15$ \\
\hline PRD-PT & & & & & & $67.845 .844,67$ \\
\hline MC & & & & & & $96.593 .640,01$ \\
\hline NA & $23,770,069.10$ & $24,152,976.31$ & $20,917,764.97$ & $56,359,631.08$ & $44,687,833$ & $80,512,607.39$ \\
\hline PH & \multicolumn{4}{|c|}{$\begin{array}{l}\text { el PH reporto egresos en el SIF por una cantidad de: } 17,437,128 \text { derivado de la verificacion } \\
\text { documental quedo como sigue } \rightarrow->\end{array}$} & & $21,321,956.06$ \\
\hline P.M. & $\begin{array}{l}\text { el PM reporto egresos en el SIF por una } \\
\text { cantidad de: } 11,896,725,+ \text { mientras que el } \\
\text { INE le ficalizo: } 20,640,574 \text {, para } \\
\text { poteriormente quedar como sigue-- } \rightarrow\end{array}$ & $8.321 .414,30$ & & $20.366 .285,93$ & & $28,687,700.23$ \\
\hline PES & \multicolumn{4}{|c|}{$\begin{array}{c}\text { el PES reporto ingresos en el SIF por una cantidad de: } 20,282,023 \text { derivado de la verificación } \\
\text { documental quedo como sigue }-\cdots\end{array}$} & & $34,672,362.64$ \\
\hline
\end{tabular}

\section{3}

DR @ 2018 . Instituto de Investigaciones Jurídicas, UNAM,

https://revistas.juridicas.unam.mx/index.php/derecho-electoral/issue/archive 
Este obra forma parte del acervo de la Biblioteca Jurídica Virtual del Instituto de Investigaciones Jurídicas de la UNAM www.juridicas.unam.mx

DOI: http://dx.doi.org/10.22201/iij_.24487910e.2016.9.10102

7. Problemas del modelo para acreditar gastos de actividades clientelares y de simulación. Menos del 10\% de gastos de operación electoral.

TABLA 7

\begin{tabular}{|c|c|c|c|}
\hline P.P. & $\begin{array}{l}\text { GASTO REPORTADO } \\
\text { CON OBSERVACIONES } \\
\text { ATENDIDAS }\end{array}$ & $\begin{array}{l}\text { GASTO ACREDITADO INE SIN OBSERVACIONES } \\
\text { ATENDIDAS }\end{array}$ & $\begin{array}{l}\text { \% QUE REPRESENTA EL GASTO QUE ACREDITA EL } \\
\text { INE, RESPECTO DELGASTO TOTAL REPORTADO } \\
\text { POR EL P.P. }\end{array}$ \\
\hline PAN & $225,640,345.18$ & $1,557,073$ & $0.69 \%$ \\
\hline PRI & $\$ 45,045,215.81$ & $1,548,810.68$ & $3.4 \%$ \\
\hline $\begin{array}{l}\text { PRI- } \\
\text { PVEM }\end{array}$ & $172,827,535$ & $13,742,32$ & $0.79 \%$ \\
\hline PVEM & $31.571 .730,37$ & $\begin{array}{l}\text { INDEPENDIENTEMEN DE LOS INFORMES PREENTADOS POR } \\
\text { EL PVEM LA UTF REPORTO: } 88,182,297 \text { A SU VEZ REPORTO } \\
\text { GASTOS DE INTER CAMPAÑA QUE BENEFICIARON LAS } \\
\text { CANDIDATURAS FEDERALES POR UN TOTAL: } 36,360,638.57\end{array}$ & \\
\hline PRD & $123,806,348.36$ & 807,787 & $0.65 \%$ \\
\hline PT & $67,745,791.15$ & $11,152,473.66$ & $16.46 \%$ \\
\hline PRD-PT & $67,845,844.67$ & $2,732,398$ & $4.02 \%$ \\
\hline MC & $96,593,640.01$ & $2,586,063.11$ & $2.67 \%$ \\
\hline NA & $80,512,607.39$ & $86,924,650.45$ & \\
\hline PH & $21,321,956.06$ & $4,279,139.44$ & $20.06 \%$ \\
\hline P.M. & $\$ 28,687,700.23$ & $316,351.50$ & $1.1 \%$ \\
\hline PES & $34,672,362.64$ & $348,508.70$ & $1 \%$ \\
\hline
\end{tabular}

8. Problemas del modelo para acreditar rebase de topes de gastos de campaña: 21 candidatos los rebasaron; sólo 6 de ellos resultaron ganadores, ninguno dentro del supuesto de nulidad de menos del 5\%, a propósito de rebases del límite de gastos (diputados federales: 1,260,000.00 pesos); pero los fiscalizadores sólo detectaron 39 rebases, y para el INE, si no los encuentra, no existen: se resiste a admitir que sí existen, pero no los detecta.

\section{TABLA 8}


Este obra forma parte del acervo de la Biblioteca Jurídica Virtual del Instituto de Investigaciones Jurídicas de la UNAM www.juridicas.unam.mx

\begin{tabular}{|l|r|r|}
\hline P.P. & \multicolumn{2}{|c|}{ Ingresos totales reportados de origen ilicito acreditados } \\
\hline PAN & $231,234,306.93$ & 0 \\
\hline PRI & $46,338,417.95$ & 0 \\
\hline PRI-PVEM & $178,017,169.45$ & 0 \\
\hline PVEM & $31,564,811.00$ & 0 \\
\hline PRD & $128,722,792.70$ & 0 \\
\hline PT & $81,135,487.65$ & 0 \\
\hline PRD-PT & $69,771,626.05$ & 0 \\
\hline MC & $97,731,376.37$ & 0 \\
\hline NA & $80,795,775.86$ & 0 \\
\hline PH & $21,328,850.59$ & 0 \\
\hline P.M. & $\$ 27,331,734.11$ & 0 \\
\hline PES & $39,314,255.74$ & 0 \\
\hline
\end{tabular}

\section{PROPUESTAS DE FORTALECIMIENTO}

1. Ante los remanentes de fondos públicos por parte de los partidos, ha llegado el momento de reducir los montos mediante una fórmula que garantice su independencia, pero que se ajuste a sus requerimientos reales. La bolsa es cuantiosa, puesto que la fórmula para calcularla no se diseñó desde un razonamiento objetivo, sino buscó desde su inicio equiparar al resto de los partidos a los montos de financiamiento que manejaba el PRI antes de la reforma de 1996. Esta fórmula fue modificada con la reforma de 2007, aunque paradójicamente el monto no sufrió merma alguna. La nueva ecuación estableció que la bolsa de financiamiento se obtiene al multiplicar $65 \%$ del salario mínimo vigente para el Distrito Federal por el número de ciudadanos inscritos en el padrón electoral, en lugar de la lista nominal que es el catálogo de los ciudadanos que efectivamente pueden votar. Aunque el financiamiento para gastos ordinarios se mantuvo intacto, sí se redujo $50 \%$ el de campañas presidenciales y $70 \%$ el de campañas intermedias. Sin embargo, si se considera que a partir de esa reforma el tiempo aire para transmitir spots es gratuito, en realidad los partidos aumentaron su ingreso disponible, porque la reducción de las prerrogativas fue mucho menor que el aumento del financiamiento en especie, vía tiempos en medios electrónicos. Hubo menos presupuesto líquido para campañas, pero los partidos salieron ganando. Como se ha señalado, la reforma de 2014 
Este obra forma parte del acervo de la Biblioteca Jurídica Virtual del Instituto de Investigaciones Jurídicas de la UNAM

incrementa la bolsa al homogeneizar la formula de integración en todo el país. Dentro de los factores a considerar, en una posible reformulación, serían los resultados electorales, el análisis del histórico de gasto de los partidos, la planeación obligatoria del gasto, etcétera.

2. Fortalecer el monitoreo de gasto. Toda vez que es el principal instrumento para detectar si un partido o coalición recibió recursos de fuentes prohibidas, para identificar la posibilidad de que se esté en presencia de una "doble contabilidad", o una subdeclaración, se sugiere invertir mayores recursos en estos ejercicios, ampliar su alcance, prohibir gastos en publicidad que no pueden ser monitoreados (por ejemplo, la publicidad en salas de cine) e incorporar a los organismos electorales locales y organizaciones de la sociedad civil en estas tareas. En los hechos, implica que la autoridad administrativa realice una actividad "detectivesca", pero bien calificada y bien equipada, para encontrar los fondos sutiles, difíciles de percibir. La mayoría de los gastos dejan evidencia. Sin embargo, lo más fácil es rastrear los gastos en medios de comunicación. Hasta antes de la reforma de 2007, éste era el principal espectro de monitoreo del IFE. Pero ahora se encuentra prohibida la contratación de espacios publicitarios en radio y televisión. Aún y cuando la contratación de estos espacios está prohibida a los partidos políticos, la legislación permite la de medios alternos que también representan gastos onerosos y que pueden ser investigados, como la publicidad callejera, monitoreo que debe complementarse con la realización de un programa de verificaciones realizado por los propios auditores del órgano. Asimismo, este tipo de ejercicios puede ampliarse para la revisión de otro tipo de gastos como pantallas electrónicas, pantallas de cine o espectaculares. Relevante resultó el monitoreo de propaganda en espectaculares, llevada a cabo por la Unidad durante el proceso 2012, georreferenciando con GPS, lo anterior con base en el artículo 227 del reglamento de fiscalización.

3. A efecto de atajar las subdeclaraciones y la alteración de documentos, resulta conveniente promover una mayor transparencia y acceso a la información de manera concurrente a las campañas. Lo anterior implica obligar a los partidos a difundir o divulgar la información financiera que presentan al INE en su portal de internet durante el desarrollo de las campañas mismas y no hasta que el Consejo General del INE haya aprobado los dictámenes respectivos, para permitir el escrutinio ciudadano oportuno y crítico, y prevenir la alteración de información. El hecho de que la 
Este obra forma parte del acervo de la Biblioteca Jurídica Virtual del Instituto de Investigaciones Jurídicas de la UNAM www.juridicas.unam.mx

ciudadanía y los medios de comunicación tengan acceso a la información sobre ingresos y gastos de los partidos, aumenta la probabilidad de que las irregularidades salgan a la luz pública. La presión de la opinión pública y la exposición de los actores políticos y donadores involucrados puede ser un poderoso factor de disuasión. Por supuesto, las reglas de transparencia de la información financiera no son libres de tensión con el derecho de participación y asociación de los individuos, ya que, al hacer públicos los nombres de las personas y montos donados, se está dando una invasión a la vida privada de las personas, pero los beneficios son mayores. ${ }^{5}$ La publicidad oportuna del financiamiento de la política trae muchos beneficios a la calidad del proceso democrático: mejora la información para el elector; facilita la identificación de vinculaciones entre políticos y sectores de interés; genera incentivos para evitar conductas corruptas y aumenta sus costos; permite la verificación del cumplimiento de las normas sobre financiamiento; pone obstáculos a la desviación de fondos públicos a favor del partido de gobierno; genera incentivos para el control recíproco de los partidos políticos, y brinda insumos y parámetros para la evaluación de las decisiones de los funcionarios electos. ${ }^{6}$ Saber quién está detrás de los candidatos o detrás de los partidos políticos, permite descubrir la coherencia del discurso político, la coherencia de la conducta de los candidatos y de los partidos políticos en sí. Finalmente, la transparencia apunta a hacer más altos los costos de las actitudes o conductas corruptas. Cuando hay transparencia es más alto el riesgo que corren los candidatos, o los funcionarios, y por lo tanto genera incentivos para un ámbito de juego en el proceso político más limpio y equitativo. En países como Costa Rica y Argentina se prevé esta divulgación de información relevante antes de la jornada electoral, mediante informes preliminares. Por supuesto que al ser preliminar, esta información se complementa con el informe final posterior al desarrollo de la elección. Dicho informe no es para auditarse. Ello se realiza una vez presentado el informe final. La vía para hacer la divulgación de esta información es internet. La divulgación de información preliminar de gastos tiene como finalidad propender al voto informado

\footnotetext{
5 Peschard, Jacqueline, Transparencia y partidos políticos, México, IFAI, 2005, pp. 38-42, disponible en: http://mail.lapaz.gob.mx/transparencia/pdfs/08.pdf.

${ }^{6}$ Ferreira Rubio, Delia, “Financiamiento político: rendición de cuentas y divulgación”, en Griner, S. y Zovatto, D. (eds.), De las normas a las buenas prácticas. El desafío del financiamiento político en América Latina, San José, Costa Rica, OEA-IDEA, 2004.
} 
Este obra forma parte del acervo de la Biblioteca Jurídica Virtual del Instituto de Investigaciones Jurídicas de la UNAM www.juridicas.unam. $\mathrm{mx}$

del elector. Esta publicidad, en la medida en que sea completa y oportuna, genera incentivos para que los partidos se controlen recíprocamente y ajusten su conducta a los parámetros legales y a las exigencias de la opinión pública. ${ }^{7}$

4. Ajustar los plazos de los procedimientos para garantizar la exhaustividad de los mismos en consonancia con los plazos del Tribunal para resolver impugnaciones. Como se ha expuesto, los plazos de presentación de informes financieros y su respectiva revisión correspondían con los tiempos establecidos para que los resultados pudiesen incidir en la calificación de las elecciones, por lo que partidos y candidatos tenían fuertes incentivos para no cumplir con la norma, pues generalmente cuando se llegaba a detectar y sancionar la conducta violatoria, la misma ya había rendido los beneficios esperados. Con la reforma se buscó acelerar los plazos, de tal suerte que los resultados del ejercicio de fiscalización sirvan para calificar elecciones y eventualmente anular las que hayan rebasado topes de gasto. La fiscalización se pudo concluir en tiempo justo para que el Tribunal Electoral del Poder Judicial de la Federación (TEPJF) pudiera resolver las impugnaciones correspondientes a más tardar el 3 de agosto. Sin embargo, la carga de trabajo obligó al INE a aplazar la aprobación de los dictámenes correspondientes a la fiscalización de gastos de campaña de candidatos federales y locales, y a que éstos no fueran exhaustivos porque no contemplaron la resolución de procedimientos de queja relacionados a posibles rebases de topes de gasto. ${ }^{8}$ Evidentemente se requiere modificar los reglamentos de fiscalización para cambiar procedimientos y fechas en materia de

\footnotetext{
${ }^{7}$ La información debe incluir varios aspectos, como el nombre del receptor de los fondos, el monto entregado, la fecha y el concepto por el cual se produce el gasto. La exigencia de identificar a los proveedores de bienes y servicios con indicación detallada de los montos correspondientes. Deben incluirse los gastos del partido y el candidato, pero también de los grupos de apoyo vinculados a éstos, puesto que generalmente se utilizan formas jurídicas para terciar fondos, para distraerlos o para generar canales ocultos de transmisión del dinero, como las fundaciones, algunas asociaciones, empresas de los partidos o lo que se llama "amigos" del candidato para evadir el cumplimiento de la ley y el control de la autoridad electoral. En consecuencia, deben ser sujetos obligados todos los actores políticos relevantes, partidos, candidatos, donantes.

8 El INE revisó 24,230 informes de campaña correspondientes a 13,550 candidatos (2,667 federales y 10,883 locales). En 2012, el entonces IFE sólo revisó 1,605 informes correspondientes a igual número de candidaturas de la elección federal. No sólo revisa más, sino en menor tiempo: en 2012 dispuso de varios meses, y en 2015 de sólo 40 días después de la jornada electoral.
} 
Este obra forma parte del acervo de la Biblioteca Jurídica Virtual del Instituto de Investigaciones Jurídicas de la UNAM www.juridicas.unam.mx

fiscalización, para que éstos puedan ser exhaustivos y coincidentes con los plazos del TEPJF para resolver impugnaciones. La Sala Superior revocó siete expedientes del INE por no investigar con exhaustividad sobre los gastos de campaña de diputados federales, y no permitir determinar si hubo o no rebase de topes de campaña. De acuerdo al TEPJF, el INE debió resolver los procedimientos de quejas antes de emitir dictámenes de fiscalización, y que no fue exhaustivo en la valoración de las pruebas. Esto impidió que los partidos políticos pudieran impugnar en primera instancia ante las salas regionales, y después, en reconsideración ante la Sala Superior. Es necesario que los asuntos relativos a la fiscalización deban quedar resueltos antes del 3 de agosto, para que sean tomados en cuenta al resolver los juicios de inconformidad, que es en donde en principio deben valorarse.

5. La homologación nacional de la fórmula para determinar los montos de topes de gasto. Como resultado de la última reforma electoral, ahora el INE fiscaliza todas las campañas del país, presuntamente para mejorar el control de los gastos ejercidos y garantizar la equidad en las contiendas. Sin embargo, con al artículo 116 de la Constitución federal, los organismos públicos locales electorales son competentes para determinar los montos de los topes de gasto en elecciones locales. Lo anterior provoca que el INE debe fiscalizar el cumplimiento de normas distintas, lo cual atenta contra el principio de igualdad, y abre la posibilidad que en algunas elecciones a gobernador o municipales se establezcan topes que no obedezcan a una lógica objetiva o racional, sino sea el saco a la medida de algún aspirante.

6. Incentivar el cumplimiento de las reglas, incrementando considerablemente los montos de los topes de gasto. El tope para cada uno de los candidatos que buscaban ser diputados federales se estableció en 1.2 millones de pesos (mdp). Con el propósito de estar preparado para fiscalizar el cumplimiento de los topes de gasto de las miles de campañas que se realizaron este año, en el INE se destinaron, según el Acuerdo CG 341/2014, \$670.7 mdp para "administrar las prerrogativas de los partidos políticos y fiscalizar sus recursos". Además, la Unidad Técnica de Fiscalización contó con un presupuesto de $\$ 244.3 \mathrm{mdp}$, para tener un programa contable de fiscalización en línea. A pesar de lo anterior, el INE afirmó que de entre los más de doce mil candidatos federales y locales sólo 39 rebasaron los topes de gasto establecidos. Pero eso no implica que efectivamente hayan sido los únicos. La percepción empírica es que hay montos importantes de recursos invertidos en las campañas electorales que 
Este obra forma parte del acervo de la Biblioteca Jurídica Virtual del Instituto de Investigaciones Jurídicas de la UNAM www.juridicas.unam.mx

no se reportan por el temor a rebasar los topes tan menores. Si se quiere incentivar el cumplimiento de las reglas, los topes tienen que incrementarse considerablemente y ajustarse a la realidad de cada elección.

7. Incentivar el cumplimiento de las reglas al permitir, bajo determinadas circunstancias, las aportaciones de personas morales con fines mercantiles o comerciales. La reforma no alteró el modelo sobrerregulado y restrictivo en materia de fuentes de financiación, sino buscó fortalecerlo y hacerlo más eficiente. El problema es que en sistemas como el nuestro, en que coinciden campañas prolongadas y precampañas adelantadas con reglas de aportaciones privadas con fuertes limitaciones y restricciones, se tiende al ocultamiento de información y a la simulación. Luego entonces, la clave para generar estímulos a los partidos y candidatos para que transparenten realmente sus fondos pasa por quitar algunos candados al financiamiento privado. Es más importante, desde el punto de vista de la calidad del proceso democrático, la transparencia sobre el origen y destino de los fondos con que los partidos y candidatos financian su actividad, que el establecimiento de límites y restricciones de difícil aplicación y control. Las restricciones severas frecuentemente acarrean consecuencias negativas para la transparencia electoral e incentiva prácticas de financiamiento político particularmente oscuras y cuestionables. ${ }^{9}$ Entre más difícil se haga a los partidos y candidatos recaudar fondos por vías legales, más probable será que recurran a procedimientos oscuros y cuestionables, y por lo mismo, que no los reporten. Más importante que poner todos estos límites, es generar incentivos de transparencia y divulgación de información oportuna de los gastos de campaña. Ante ello no debemos cerrarnos a la posibilidad de que se permita, por ejemplo, que personas morales con fines de lucro puedan aportar a través de depósitos bancarios y cheques, en aras de una democracia más confiable e, irónicamente, menos onerosa para la ciudadanía. Lograrlo implicaría romper con mitos y prejuicios hacia las contribuciones privadas que, en general, confunden más de lo que iluminan, y distorsionan más de lo que describen. ${ }^{10}$ Sobre todo en un país cuya transición democrática ha sido posible en

\footnotetext{
9 Levush, Ruth, Compaign Financing of National Elections in Foreign Countries, Washington, D. C., Law Library of Congress, 1991, pp. 90-92; Alexander, Herbert et al., New Realities, New Thinking: Report of the Task Force on Campaign Finance Reform, Citizens' Research Foundation-University of Southern California, 1997.

10 Casas Zamora, Kevin, “Regulando el financiamiento político en Costa Rica: Algunas reflexiones prácticas”, en Prado, Mimi (ed.), Modernización del Estado costarricense, San José de Costa Rica, Centro Internacional
} 
Este obra forma parte del acervo de la Biblioteca Jurídica Virtual del Instituto de Investigaciones Jurídicas de la UNAM www.juridicas.unam.mx

buena medida por el éxito en establecer cuantiosos y equitativos fondos públicos, y por establecer límites y prohibiciones a la financiación privada. El éxito del modelo ha generado que actualmente se idolatre y que las propuestas de liberar la financiación privada se consideren un sacrilegio. Nadie discute que el financiamiento mixto que limitaba los fondos privados resultaba necesario en un contexto determinado. La reforma electoral de 1996 favoreció el acceso de los partidos de oposición a un subsidio estatal excepcionalmente generoso, que contribuyó a la emergencia de un sistema plural de partidos estable. ${ }^{11}$ Sin embargo, se ha demostrado que el financiamiento público no aleja a los grandes donadores privados de los partidos. Por lo tanto, quizá haya llegado el momento de transitar hacia un modelo más acorde con los nuevos tiempos. Mantener el modelo de financiamiento mixto, pero dando la oportunidad de que los políticos accedan a mayores fondos privados. Menores topes y prohibiciones facilitarían la transparencia de los fondos. Se generarían incentivos para que éstos sean reportados. Definitivamente, se trata de medidas que conllevan riesgos, pero me parece que al menos ha llegado la hora de quitarse los prejuicios, y lejos de evadir el debate, se abra una amplia y razonada reflexión en torno a esta posibilidad.

8. Discutir la posibilidad de implementar medidas como el voto obligatorio, para prevenir los efectos del inevitable clientelismo en contextos de marcada desigualdad socioeconómica. Una actividad que suele distorsionar los sistemas de financiación electoral formal es el ascendente clientelismo electoral por las dificultades para prevenirlo, acreditarlo y sancionarlo. Un estudio de la Universidad de Notre Dame sugiere que $38 \%$ de los votantes mexicanos recibieron algún regalo o favor a cambio del voto en la elección de 2009, la cifra más alta del estudio que incluye a países como Nicaragua, Bolivia, Uruguay y Honduras. Se trata de una actividad que no es ética, tampoco lícita, pero que a pesar de ello suele realizarse e implicar una enorme

para el Desarrollo Humano-Fundación Konrad Adenauer, 2004, pp. 254-259; Smith, B. A., Unfree Speech: The Folly of Campaign Finance Reform, pp. 39-65; Sorauf, Frank, Inside Campaign Finance: Myths and Realities, New Haven, Yale University Press, 1992, pp. 161190.

11 Woldenberg, José et al., “El modelo de financiación de los partidos políticos en México", en Castillo, P. del y Zovatto, D. (eds.), La financiación de la política en Iberoamérica, San José, Costa Rica, Instituto Interamericano de los Derechos Humanos-Capel, 1998; Sartori, G., Partidos y sistemas de partidos, Madrid, Alianza, 1991, p. 260. 
Este obra forma parte del acervo de la Biblioteca Jurídica Virtual del Instituto de Investigaciones Jurídicas de la UNAM www.juridicas.unam.mx

cantidad de recursos. La estrategia ha sido incorporada a los repertorios estratégicos de todos los partidos, los cuales echan mano de ella en cuanto tienen recursos para aplicarla. Todos los partidos se dedican a repartir materiales, despensas y dinero durante la campaña electoral, y el día de las elecciones transportan a votantes a las casillas electorales. Esto ha sido un signo común en las elecciones realizadas en estados con niveles altos de marginalidad urbana y rural. En la mayoría de esos lugares, las inconformidades más importantes en las que se basaron las pretensiones partidistas de nulidad, fueron los actos de clientelismo, en su forma delictiva de compra y coacción del voto. El peso de la operación corporativa ha disminuido en importancia, pero en su lugar ha llegado un amplio y bien aceitado esquema clientelar; los ciudadanos se reducen -en muchos casos- a beneficiarios, y el voto es una mercancía que se compra en el mercado de los intercambios de pobreza, favores, programas sociales y apoyo a candidatos que hacen campaña para comprar votos. Se debe reconocer que hay poco por hacer para fiscalizar los gastos destinados a actividades como la compra de votos, pues la solución a este problema pasa por mejorar las condiciones socioeconómicas del país. Requiere del ataque frontal a las enormes desigualdades socioeconómicas que generan condiciones propicias para la práctica de esta conducta. Requiere generar condiciones para que, a la idea de Rousseau, no haya nadie tan pobre que tenga necesidad de venderse y nadie tan rico que pueda comprar a alguien. Ante las dificultades de que eso se logre en el corto plazo, no debemos descartar la posibilidad de incorporar medidas que resten rentabilidad electoral a las prácticas clientelares, siendo el voto obligatorio una de las medidas que mejor podría desincentivar estas prácticas.

Finalmente, debemos reconocer que los esfuerzos para mejorar la calidad de las reglas de financiamiento político-electoral son insuficientes si no se asume un auténtico compromiso republicano por parte de todos los actores involucrados en el mercado. Se requieren actores políticos a quienes les importe el juicio de la historia: el cómo ganan. Interesados en el juego limpio. Pero también el reto requiere más ciudadanía en la política, más ciudadanía participando, exigiendo y actuando para construir también partidos políticos más abiertos y transparentes, más responsables. Una prensa de investigación más interesada en estos temas. Una de las razones por las cuales los ciudadanos no se involucran en estas actividades es la poca valoración de la transparencia. La gente no necesariamente castiga con su voto al partido poco 
Este obra forma parte del acervo de la Biblioteca Jurídica Virtual del Instituto de Investigaciones Jurídicas de la UNAM www.juridicas.unam.mx https://biblio.juridicas.unam.mx/bjv

https://revistas.juridicas.unam.mx/

DOl: http://dx.doi.org/10.22201/iij.24487910e.2016.9.10102

transparente, sino que vota por otras motivaciones. Es importante que los ciudadanos no seamos displicentes y rechacemos candidatos que no cumplan con sus obligaciones en materia de transparencia durante las campañas. Mientras nosotros no le asignemos a la transparencia un valor central en las decisiones políticas de una sociedad, va a ser difícil que modifiquemos la reacción de los políticos y de los partidos políticos.

\section{3}

DR @ 2018. Instituto de Investigaciones Jurídicas, UNAM, https://revistas.juridicas.unam.mx/index.php/derecho-electoral/issue/archive 